\title{
PERCEPCIÓN DE CONGESTIÓN Y DIMENSIÓN SOCIAL DE LA CAPACIDAD DE CARGA EN CENOTES DE YUCATÁN
}

\author{
Fernando Enseñat-Soberanis* \\ Universidad de Quintana Roo/Universidad Autónoma de Yucatán \\ https://orcid.org/0000-0002-6798-7407 \\ Rocío Blanco-Gregory** \\ Universidad de Extremadura \\ https://orcid.org/0000-0002-0105-5535 \\ Johnathan Alexander Mondragón-Mejía*** \\ Universidad Nacional Autónoma de México \\ https://orcid.org/0000-0001-6564-7065
}

\section{RESUMEN}

La visita masiva de turistas a los cenotes de Yucatán tiene impactos negativos sobre dos elementos: el recurso natural y la experiencia misma del visitante. Este estudio mide y compara, a través del indicador percepción de congestión, los impactos de la masificación en la experiencia del visitante en dos cenotes de Yucatán y entre tres tipos de visitantes: locales, nacionales y extranjeros. Los resultados muestran que la aceptabilidad de los visitantes a la congestión disminuye conforme aumenta el número de personas en ambos cenotes. Los visitantes extranjeros en los dos cenotes toleran menos la congestión mientras que los locales son el grupo que presentan niveles de tolerancia más altos.

Palabras clave: Impactos sociales del turismo; ecoturismo; estándares de congestión: capacidad de carga de visitantes.

Fecha de recepción: 14 de enero de 2019.

Fecha de aceptación: 15 de julio de 2019.

* Doctorado en Desarrollo Sostenible. División de Desarrollo Sostenible (DSS).Universidad de Quintana Roo. Avenida Andrés Quintana Roo s/n, Colonia San Gervasio. 77600 COZUMEL, Quintana Roo (México) y Facultad de Ciencias Antropológicas, Universidad Autónoma de Yucatán, Km. 1, carr. Mérida-Tizimín, MÉRIDA, Yucatán (México). E-mail: fernandoensenat@gmail.com

** Departamento de Dirección de Empresas y Sociología. Facultad de Empresa, Finanzas y Turismo. Universidad de Extremadura. Avda. de la Universidad, s/n. 10076 CÁCERES (España).E-mail: rblanco@unex.es

*** Unidad Multidisciplinaria de Docencia e Investigación. Facultad de Ciencias. Universidad Nacional Autónoma de México (Campus Sisal). Puerto de Abrigo, s/n. 97356 SISAL Yucatán (México). E-mail: johnybc123@gmail.com 


\title{
Perception of congestion and social dimension of the carrying capacity in Yucatan cenotes
}

\begin{abstract}
The massive visit of tourists to the cenotes of Yucatán has negative impacts on two elements: the natural resource and the visitor's own experience. This study measures and compares, through the congestion perception indicator, the impacts of massification on the visitor's experience in two cenotes of Yucatán and among three types of visitors: local, national and foreign. The results show that the acceptability of visitors to congestion decreases as the number of people in both cenotes increases. Foreign visitors in the two cenotes tolerate less congestion while locals are the group with the highest tolerance levels.
\end{abstract}

Key words: Social impacts of tourism; ecotourism; congestion standards; social dimension; carrying capacity of visitors.

\section{INTRODUCCIÓN}

Los impactos que el exceso de visitantes está causando en áreas naturales pueden ser divididos en ecológicos y sociales. Los ecológicos incluyen compactación del suelo, pérdida de cobertura vegetal, contaminación del aire, del agua y auditiva (Manning y Anderson, 2012; Leung y Marion, 1999), así como estrés de la fauna silvestre (Newsome, Dowling y Moore, 2005). Los sociales incluyen percepción de congestión y percepción de daño ambiental por parte de los visitantes, satisfacción y comportamiento inadecuado del visitante, así como conflictos entre diferentes tipos de visitantes que usan un mismo espacio natural (ej. caminantes y ciclistas usando un mismo sendero en un parque nacional) (Tyler, Roberts y Nilsen, 1997).

En los países menos desarrollados, la visita de locales y turistas extranjeros en ciertos espacios naturales es considerada como una estrategia económica que contribuye a la conservación y a la generación de ingresos para las comunidades rurales que viven alrededor (Drumm y Moore, 2002). Para el caso de la península de Yucatán, al igual que muchas regiones de México y Latinoamérica, los pobladores rurales han subsistido practicando diversas actividades económicas como son la agricultura, la ganadería, la caza, la pesca, el aprovechamiento de productos maderables y no maderables, entre otros. Aunado a estas actividades, en las últimas décadas, el turismo comenzó a ser una actividad importante para algunos campesinos de la península que vieron en esta actividad un complemento significativo a sus precarios ingresos (Blanco, Enseñat y Mondragón, 2019).

Los cenotes de la península de Yucatán son cuevas inundadas de agua dulce y forman una de las reservas hidrológicas más grandes de México. Aunque la mayoría de los cenotes aún son usados para fines agrícola-ganaderos, de 2014 a 2018 treinta y dos nuevos cenotes fueron registrados con algún tipo de uso turístico-recreativo (SEDUMA, 2018; SEFOTUR, 2017). Este rápido cambio en el uso de los cenotes está vinculado al exponencial crecimiento económico y urbano de ciudades como Mérida y de regiones turísticas como 
Cancún-Riviera Maya y su traspaís (Jouault \& Jiménez, 2015). Sin embargo, como sucede con muchas atracciones turísticas, este crecimiento no es homogéneo y existen cenotes que están ganando rápidamente visitantes, mientras otros los están perdiendo (García de Fuentes, Jouault, \& Romero, 2015; McKercher \& Du Cros, 2002).

Los cenotes Dzombakal y X’batún se encuentran en el Ejido de San Antonio Mulix, en el estado mexicano de Yucatán. Del total de cenotes registrados en el Estado de Yucatán, el 56\% se encuentran en ejidos (SEDUMA, 2018). Los ejidos son tierras de uso común, localizados en su mayoría en zonas rurales y cuya propiedad recae, en muchos casos, en grupos indígenas.

Así, una gran parte de los cenotes son administrados por cooperativas ecoturísticas de campesinos mayas que, como organizaciones de la economía social, tienen un doble desafío: por un lado necesitan mantener o incrementar el ya de por sí alto número de visitantes que llegan a sus cenotes porque representa una fuente complementaria de ingresos para los socios y sus familias (Jouault, 2018); y por otro, deben cumplir con la normatividad medioambiental vigente que les obliga a contar con un estudio de capacidad de carga que limite el uso del cenote en favor de su conservación (SEDUMA, 2014). Aunque en el Artículo $4^{\circ}$, fracción VIII del Reglamento de la Ley de Protección al medio ambiente del Estado de Yucatán en materia de cenotes, cuevas y grutas (SEDUMA, 2014) se establece que es competencia del poder ejecutivo del Gobierno del Estado de Yucatán establecer la metodología para la elaboración del estudio de capacidad de carga turística, esta metodología no ha sido aún determinada. Así, el presente estudio tiene dos objetivos: 1) Contribuir a la construcción de una metodología para estimar la capacidad de carga turística en los cenotes de Yucatán, a través de la identificación de los estándares de congestión y 2) Comparar estos estándares entre tres tipos de visitantes según su origen local, nacional e internacional.

\section{DIMENSIÓN SOCIAL DE LA CAPACIDAD DE CARGA DE VISITANTES}

El exceso de uso de un recurso por parte de los visitantes en un área natural tiene impactos principalmnete sobre dos elementos: la naturaleza y la experiencia del visitante. En un principio, la atención se centró unicamente en los impactos a la naturaleza tratando de cuantificar cuántos visitantes es demasiado, surgiendo así, las primeras aplicaciones de la capacidad de carga en sitios turistico-recreativos.

Fue en los parques nacionales de Estados Unidos en la decada de 1960 cuando se dieron los primeros estudios de capacidad de carga en áreas de uso recreativo. Estos estudios buscaban establecer el número máximo de visitantes que un parque podía recibir antes de que el recurso natural comenzara a dañarse (Manning \& Anderson, 2012). Con el paso del tiempo se comprendió que la disminución de los impactos ecológicos solo puede lograrse mediante la consideración de los impactos sociales, tal y como lo ilustra este pasaje de Wagar (1964: 23):

“Este estudio fue iniciado pensando que la capacidad de carga en sitios recreativos podía estimarse, principalmente, considerando solo el deterioro a la naturaleza y 
la ecología. Sin embargo, pronto se volvió evidente que este punto de vista debía ser completado con la consideración de valores humanos" ${ }^{.}$.

Así, los impactos a la experiencia del vistante se incorporan como un elemento fundamental para disminuir los impactos ecológicos ya que ambos elementos están interrelacionados y son dos caras del mismo concepto. En otras palabras, no hay conservación sin la gente y sólo incorporando indicadores sociales en el manejo de los sitios se podrá atacar de manera efectiva los impactos a la naturaleza (Figura 1).

\section{Figura 1 \\ DIMENSIONES ECOLÓGICA Y SOCIAL DE LA CAPACIDAD DE CARGA DE VISITANTES}

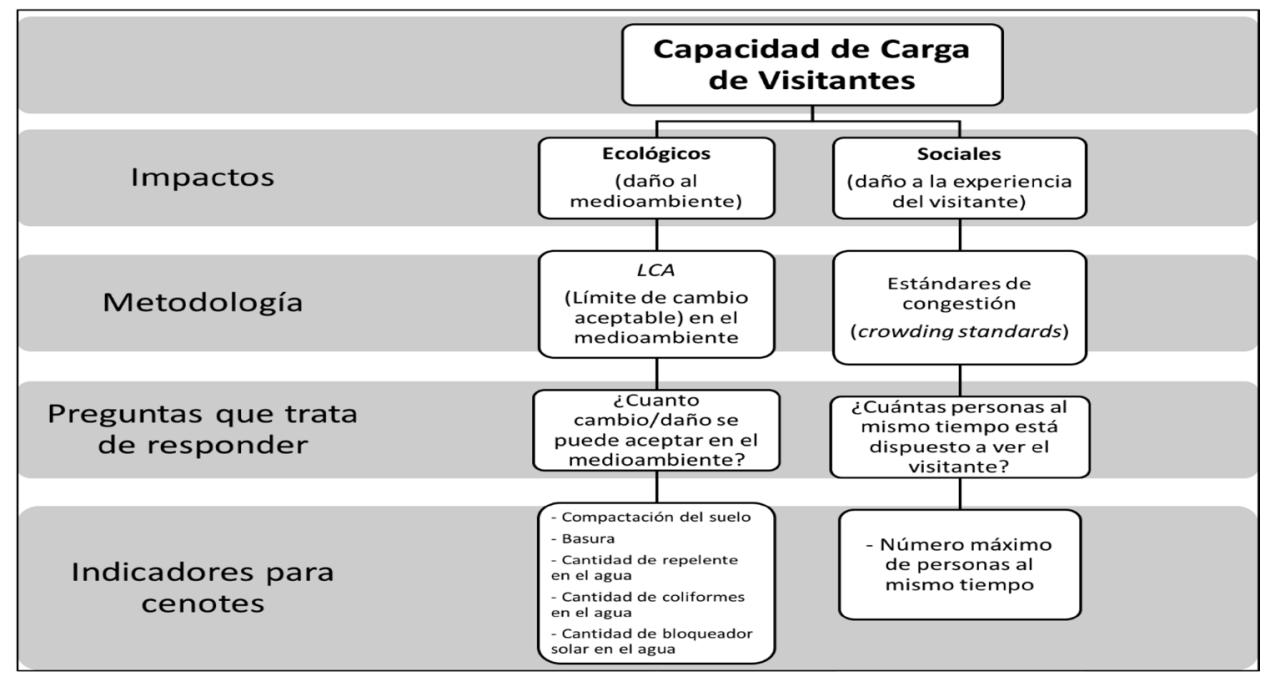

Fuente: Elaboración propia.

\section{PERCEPCIÓN DE CONGESTIÓNY CAPACIDAD DE CARGA}

La percepción de congestión o multitud de personas en los lugares de recreación al aire libre es definida por muchos autores como una evaluación negativa de la densidad de población en un lugar concreto e implica un juicio de valor, por parte del turista, de que la densidad o el número de encuentros simultáneos con otros visitantes son demasiados (Vaske \&Shelby, 2008). En esta misma línea, estos autores argumentan que el término "congestión percibida" o crowding (en inglés) se usa, a menudo, para enfatizar la naturaleza subjetiva que posee dicho concepto. El concepto de congestión está fuertemente asociado a los de capacidad de carga perceptual y capacidad de carga psicosocial. La primera se refiere al número máximo de individuos que un turista o visitante está

1 Traducción libre del original. 
dispuesto a ver en un área determinada antes de que su nivel de satisfacción comience a decrecer y la segunda, además de determinar un número máximo de personas que se tolera ver, analiza el nivel de conflicto por diferentes usos que se le puede dar a un mismo espacio natural (ej. ciclistas y corredores que usan un mismo sendero dentro de un Parque Nacional) (Burns, Arnberger y Von Ruschkowski, 2010). La capacidad de carga psicosocial es un término más amplio que considera a la capacidad de carga perceptual, además de otros conceptos.

Coccossis (2013) propone la definición de capacidad de carga del visitante como la estimación del número máximo de personas que pueden estar en un sitio sin que su presencia cause daños irreversibles en el ambiente construido y natural, y se produzca una disminución en la calidad de su experiencia. García Hernández, De la Calle Vaquero y Mínguez García (2011) proponen que la capacidad de carga de visitantes tiene, a su vez, tres dimensiones: la física, la perceptual y la administrativa. La capacidad de carga física se refiere al número máximo de personas que la superficie de un sitio puede recibir tomando en cuenta la superficie en metros o kilómetros cuadrados. La capacidad de carga perceptual es el número máximo de personas que el visitante está dispuesto a ver en un sitio turístico y la capacidad de carga administrativa se refiere al número de visitantes máximo que el personal de un sitio es capaz de manejar de acuerdo a los recursos, tanto económicos como humanos, con los que cuenta (Blanco, Enseñat y Mondragón, 2019:172).

La percepción de multitud o crowding es un indicador que toma valores llamados estándares. Los estándares son "condiciones mínimas aceptables de un indicador" (Alazaizeh et al., 2015). Son criterios utilizados para evaluar las condiciones ambientales, sociales o gerenciales de un sitio. En este sentido, las metas y objetivos de la gestión se reflejan al determinar los indicadores apropiados, y los estándares son juicios de valor cuantificables que reflejan lo que la administración está tratando de lograr. Es decir, la ventaja de establecer unos estándares (en el sentido de condiciones mínimas aceptables de carácter ambiental, social y gerencial) reside en que los equipos humanos que gestionan los lugares patrimoniales y de recreación pueden actuar de forma proactiva y no reactiva, estableciendo prioridades en la gestión de dichos sitios y anticipándose cuando los impactos físicos o sociales alcancen o superen los niveles mínimos (Vaske, 2002; Whittaker \& Shelby, 1992).

El estándar de congestión o crowding standard refleja números máximos aceptables de visitantes y su variabilidad dependerá, en gran medida, de las normas personales y sociales de cada visitante o grupo social.

\section{TEORÍA NORMATIVA Y PERCEPCIÓN DE CONGESTIÓN}

Desarrolladas en los campos de la sociología y la psicología social, las normas han atraído considerable atención como construcción teórica y marco empírico en la investigación y la gestión de actividades turístico-recreativas. Las normas se refieren a lo que se considera normal o aceptado por un individuo (normas individuales) o un grupo social (normas sociales) (Manning, 2013; Manning \& Anderson, 2012). Las normas sociales, a diferencia de las actitudes, que son evaluaciones positivas o negativas del comportamiento, tienen un carácter punitivo que sanciona el comportamiento de manera 
formal o informal (Manning, 2013). Muchas normas se convierten en política pública a través de reglas, reglamentos o leyes.

En este sentido, cuando nos referimos a los estándares de congestión que tienen los individuos a la hora de aceptar un número determinado de personas permaneciendo a la vez en el mismo lugar, hacemos referencia, por un lado, a una tolerancia individual que tiene que ver con la personalidad del individuo, y, por otro, a una tolerancia de carácter más social donde los individuos se ven influenciados por las normas sociales que rigen en el conjunto de su ámbito social y que tienen que ver con sus tradiciones, costumbres, nivel de educación, formas de comportamiento, etc., que imperan en su entorno. Estos estándares de multitud están relacionados con el término utilizado por Vaske yShelby (2008) cuando hacen referencia a la multitud percibida por una persona como una construcción psicológica que existe en las mentes de los individuos y que, generalmente, se mide mediante técnicas de auto-informe.

En esta misma línea Manning y Anderson (2012) apuntan que, si los visitantes tienen normas relativas a los aspectos de las experiencias recreativas, esas normas pueden medirse y utilizarse como base para formular normas de calidad. De esta manera, los estándares de congestión se pueden determinar y gestionar de manera más eficaz. Al respecto, hay que añadir el conflicto que se desprende de las normas sociales y las consecutivas sanciones sociales que pueden conllevar la no observación de dichas normas, pues las normas sociales pueden ser efectivas a nivel social y no tanto a nivel individual de las personas que visitan y hacen uso de las áreas recreativas, o, por el contrario, pueden ser más efectivas a la hora de regular el comportamiento individual y no el colectivo. Es conveniente señalar que, generalmente, las normas se refieren a lo que se considera normal o aceptado dentro de un grupo social. Dichas normas representan un conjunto de reglamentos y directrices que especifican cómo se espera que las personas se comporten bajo unas condiciones particulares en un lugar determinado.

En la línea de Vaske y Whittaker (2004), en psicología social, se expone que las normas se miden y se aplican fundamentalmente a través de tres paradigmas: los enfoques de normas y modelos de activación, la teoría de la acción razonada y los modelos de características estructurales. Éstos últimos se utilizan ampliamente en la formulación de normas en parques y áreas afines, y tienen como objetivo comprender las características de las normas sociales usando el Modelo de Retorno Potencial (RPM) desarrollado por (Jackson, 1966). Según Jackson (1966:36), el RPM mide la relación existente entre comportamiento y aprobación dentro de un grupo social:

"representa una definición de la norma en términos de la distribución de la aprobación y la desaprobación potencial por los demás para diferentes opciones de comportamiento mostradas por los actores a lo largo de un continuo bajo condiciones especificadas, es decir, en una determinada situación definida”.

Con el uso de esta metodología, las normas personales de los turistas pueden ser utilizadas para probar la existencia de normas sociales o el grado en que las normas son compartidas entre grupos. La investigación normativa aplicada a espacios recreativos al 
aire libre se ha centrado, en gran medida, en el problema de la congestión o saturación por visitantes (Vaske y Donnelly, 1988; Shelby, Vaske y Harris, 1988).

El Modelo de Retorno Potencial, propuesto por Jackson (1966) (Figura 2), incluye dos componentes principales: la dimensión del comportamiento (eje X) que representa un número de comportamientos posibles en una situación particular y la dimensión de evaluación (eje Y) (por ejemplo, aceptabilidad). La curva de potencial de retorno, también denominada curva de normas sociales, se representa para describir los sentimientos de los miembros del grupo sobre una dimensión específica del comportamiento en una situación concreta. Las calificaciones de actitud de los miembros individuales del grupo se promedian y sirven como base para la curva. Se pueden cuantificar varias características de la curva y, así, medir y describir diferentes atributos de las normas, tales como el intervalo de condición aceptable, la intensidad de la norma, la condición óptima o preferida, la condición mínima aceptable y la cristalización (Alazaizeh et al., 2015).

\section{Figura 2}

\section{CURVA DE LA NORMA SOCIAL}

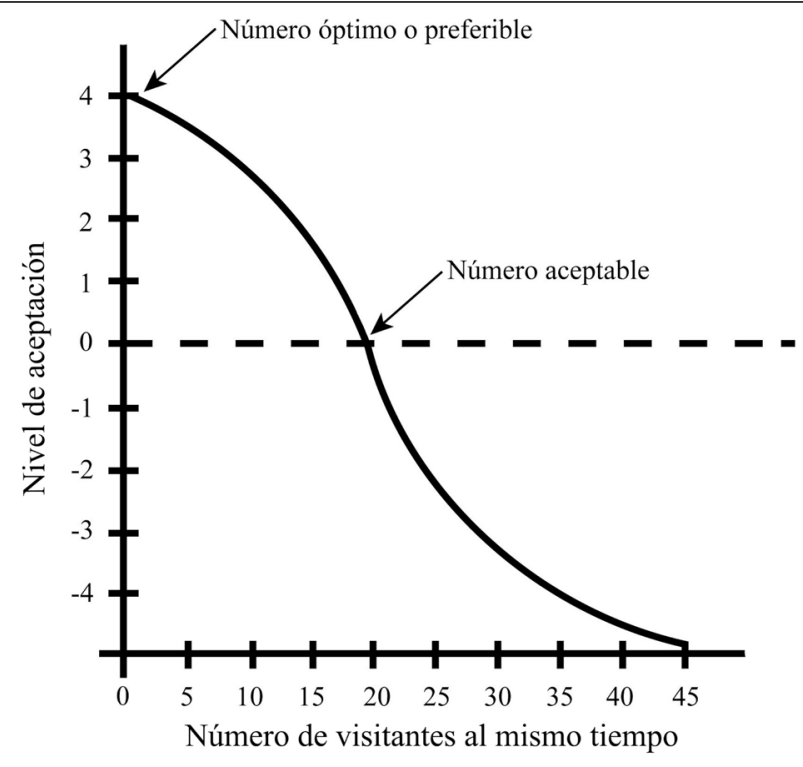

Fuente: Elaboración propia adaptado de Manning (2013).

Estas normas pueden ser estudiadas usando el RPM, pidiendo a los visitantes que realicen su evaluación sobre diferentes condiciones del sitio turístico. Siguiendo a Vaske yShelby (2008), podemos comprobar que Heberlein y Vaske (1977) desarrollaron una medida relativamente simple de la congestión percibida que solicita a las personas que indiquen cómo de concurrida estaba un área turística en el momento de su visita. Las respuestas se dan en una escala de 9 puntos: una respuesta de 1 o 2 indica que el área 
no está abarrotada; entre 3-4 indica que tiene un poco de gente; de 5 a 7 indica que está moderadamente concurrida; y de 8 a 9 indica que está muy concurrida. La medida de aglomeración de un solo ítem es fácil de completar e interpretar y ha sido ampliamente utilizada en la investigación de recreación al aire libre (Shelby et al., 1989). Hasta hace diez años, este tipo de medida se había utilizado en, al menos, 181 estudios diferentes.

Por otro lado, existen dos métodos para determinar los estandares de multitud (crowding standars) en áreas naturales. Por un lado, un enfoque narrativo y numérico tradicional (Shelby y Heberlein, 1986), y, por otro, un enfoque visual (Manning, Freimund, Limey Pitt, 1996). Ambos enfoques se basan en pedir a los visitantes y/o turistas que evalúen la aceptabilidad sobre el número de personas que están dispuestos a ver al mismo tiempo en un sitio determinado. Sin embargo, el enfoque visual ha demostrado ser más efectivo en sitios con alto aforo en donde es dificil que el vistante recuerde con precisión el número de personas que vió (Manning, Freimund, Limey Pitt, 1996).

Profundizando aún más en este tipo de medida, gran parte de la literatura existente sobre la aplicación de la curva de la norma social se centra principalmente en dos puntos para formular estándares de calidad (Manning, Freimund, Lime y Pitt, 1996). El primer punto se define como el nivel más alto a lo largo de la curva, y podría denominarse el óptimo o preferido. El segundo punto se define como el punto en el que la curva de la norma cruza desde el rango de aceptabilidad hasta el rango de inaceptabilidad. Esto podría llamarse el estándar mínimo de calidad.

\section{5. ÁREA DE ESTUDIO: CENOTES DZOMBAKAL Y X'BATÚN EN SAN ANTO- NIO MULIX, YUCATÁN, MÉXICO}

Siguiendo a Blanco, Enseñat y Mondragón (2019), los cenotes son ecosistemas característicos de la península de Yucatán y comparten rasgos únicos que permiten el desarrollo y sustento de la biodiversidad y de los asentamientos humanos circundantes. La palabra cenote se castellanizó del vocablo maya "ts'onot" que significa "caverna con depósito de agua" (Beddows et al., 2007). Este término abarca toda manifestación kárstica que alcance el nivel freático, es decir, cualquier espacio subterráneo con agua dulce con la condición de contar con una abertura al exterior, sin importar su diámetro (Gaona-Vizcayno et al., 1980).

Los cenotes de Dzombakal y X’batún se encuentran dentro del Ejido de San Antonio Mulix, a 49 km de la ciudad de Mérida, capital del estado de Yucatán (Figura 3).

Según datos oficiales, la población del Ejido San Antonio Mulix pasó de 44 a 33 habitantes de 1990 a 2009 (INEGI, 1991; SEDESOL, 2013). El 64 \% de la población se dedica al ecoturismo organizado bajo la forma de dos cooperativas: Xuux Ek y Tumben Zazil Kin Dzonot. Ambas cooperativas ofrecen como atractivo principal el uso de los cenotes, además de prestar servicios de hospedaje en cabañas rústicas y gastronomía en dos restaurantes. Se ofrece también renta de bicicletas y equipo para hacer sonorkel y para hacer campismo. La cooperativa Tumben Zazil Kin Dzonot, además de ofrecer los servicios turísticos ya mencionados, es la encargada de administrar el uso turístico de los cenotes de Dzombakal y X’batún. El ecoturismo representa la principal fuente de ingreso de la población, ya que se cobra una tarifa de entrada por su uso y disfrute. En los últimos años ha aumentado de manera significativa el número de visitantes a ambos cenotes, 
registrando entre 2015 y 2016 un incremento del $12 \%$, sobre todo de visitantes de fin de semana provenientes de la ciudad de Mérida y, en menor medida, de visitantes de otros estados de México, así como de otros países.

\section{Figura 3 \\ MAPA CON LOS CENOTES DZOMBAKAL Y X'BATÚN}

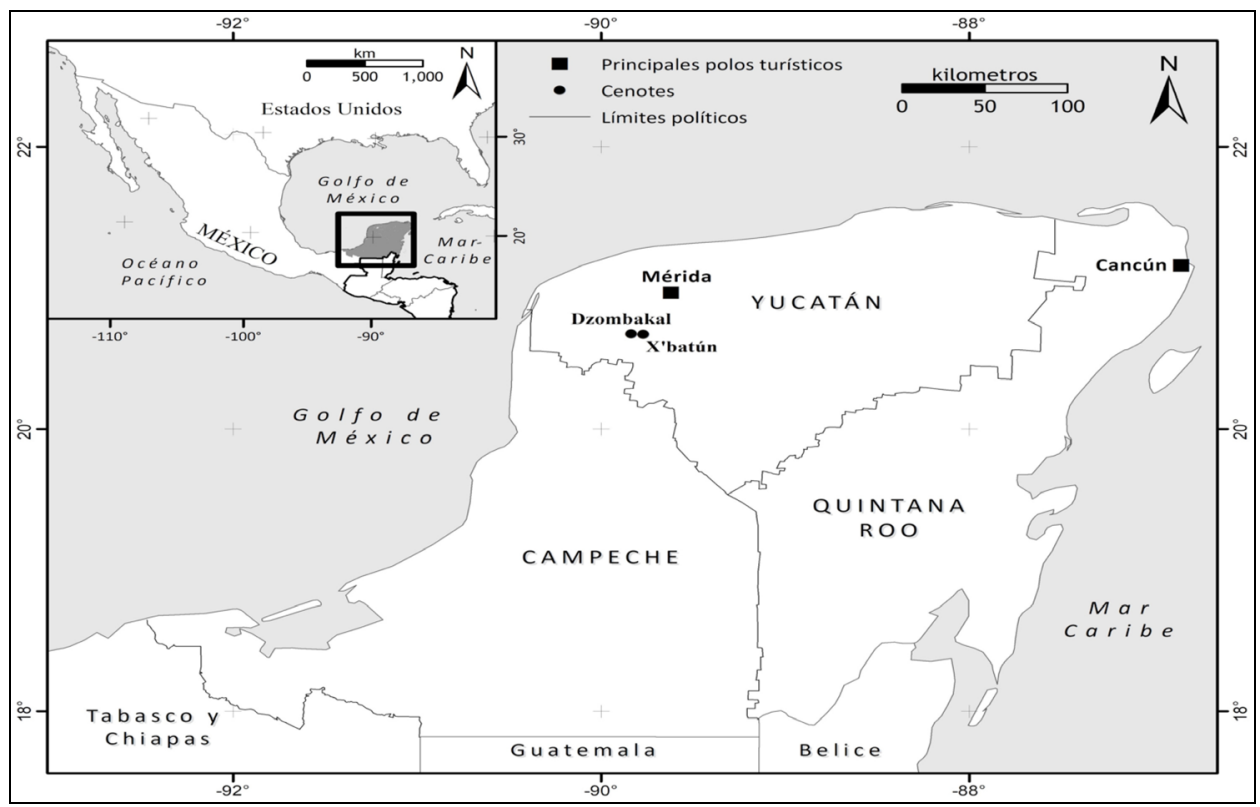

Fuente: Elaboración propia.

\section{DISEÑO METODOLÓGICO}

Para recolectar los datos de este estudio se diseñó un cuestionario a nivel cuantitaivo basado en la teoría normativa. Los cuestionarios fueron administrados, cara a cara, a una muestra representativa de visitantes de los cenotes Dzombakal y X’batún en la temporada alta de vacaciones de abril del 2017. Las muestras son mutuamante excluyentes (los visitantes encuestados en un cenote son diferentes a los encuestados en el otro). Se registró el porcentaje de visitantes que accedieron responder al cuestionario y los datos fueron procesados usando el programa SPSS Statistics versión 24.

El cuestionario está dividido en dos partes. La primera incluye datos socidemográficos generales, entre los que se encuentra el lugar de residencia que permitió clasificar a los ecoturistas en locales, nacionales e internacionales. La segunda parte incluye un conjunto de preguntas para comprender la aceptación de los visitantes a la multitud (crowding acceptability) usando una escala de 9 puntos desarrollada por Heberlein y Vaske (1977). Se utilizó el método visual debido a los elevados niveles de uso que ambos cenotes presentan. 
Se pidió a los visitantes de cada cenote evaluar el nivel de aceptación de la multitud en una escala de 9 puntos (de +4 a -4) para una serie de 6 fotografías con diferente número de personas cada una (Figura 4 y Figura 5).

\section{Figura 4 \\ FOTOGRAFÍAS DEL CENOTE X’BATÚN UTILIZADAS PARA EL ESTUDIO}

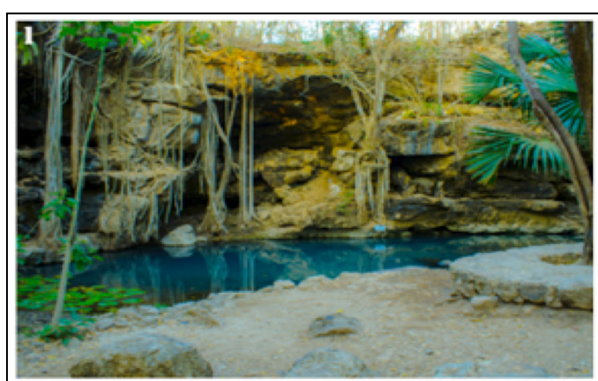

Fotografia 1: 0 personas

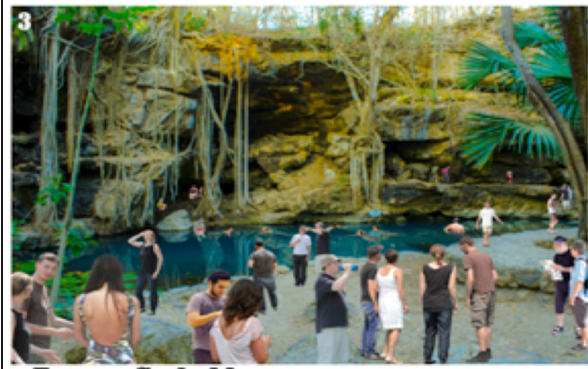

Fotografia 3: 32 personas

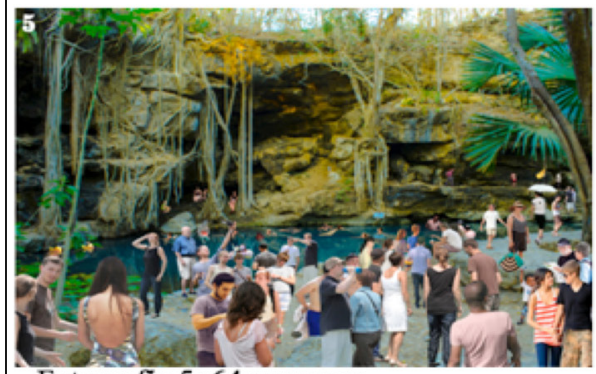

Fotografia 5: 64 personas

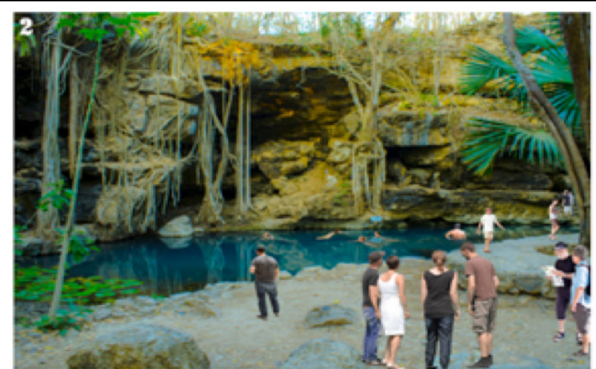

Fotografia 2: 16 personas

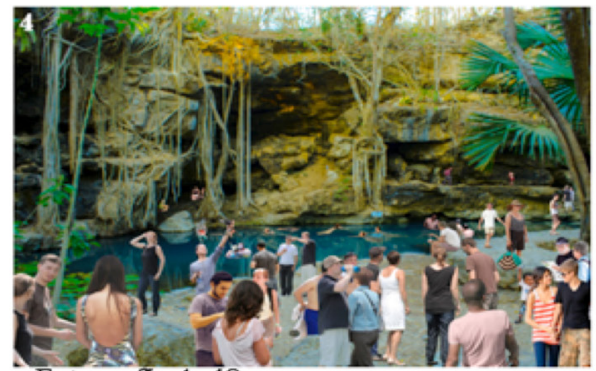

Fotografia 4: 48 personas

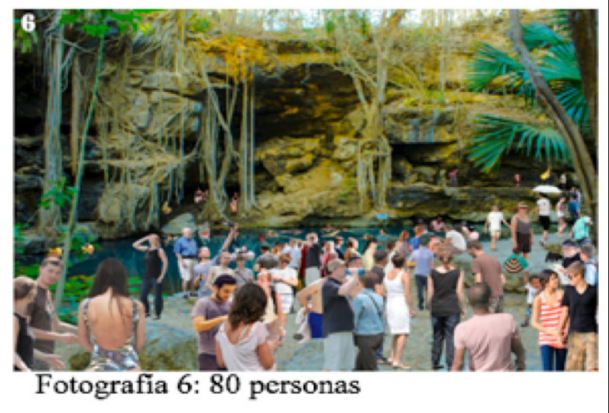

Fuente: Elaboración propia. 


\section{Figura 5 \\ FOTOGRAFÍAS DEL CENOTE DZOMBAKAL UTILIZADAS PARA EL ESTUDIO}

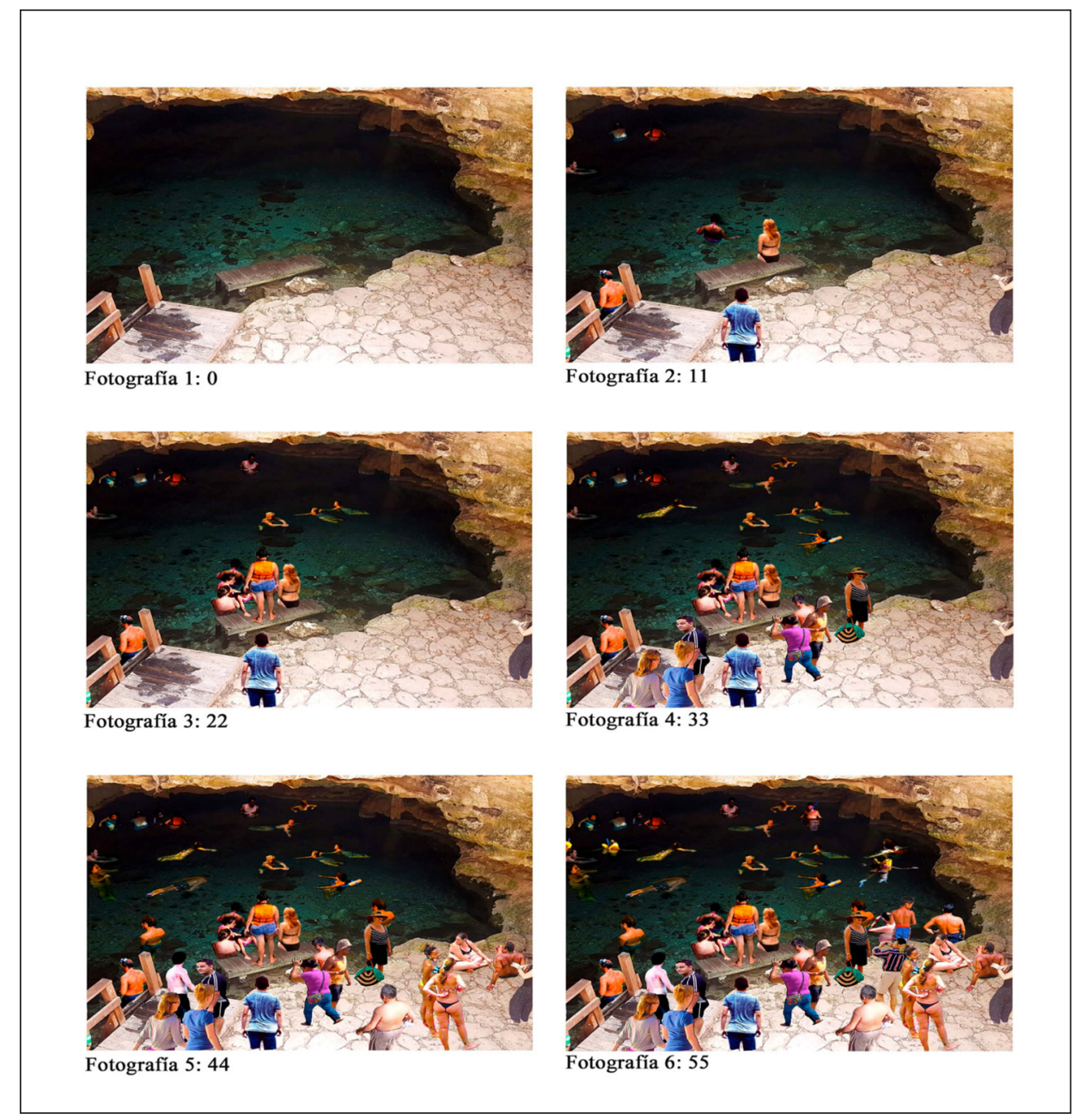

Fuente: Blanco, Enseñat y Mondragón (2019).

Las fotografías fueron manipuladas con el programa Photoshop Ps6, de manera que se obtuvieran 12 imágenes diferentes, 6 de cada cenote. La primera imagen de cada 6 , tenía cero visitantes y la última tenía el número equivalente a la densidad máxima de uso del cenote. Es decir, la cantidad máxima de personas al mismo tiempo que cada cenote puede albergar de acuerdo a su superficie de agua (Figura 6 y Figura 7) y área de descanso, en metros cuadrados. Se consideró la densidad máxima de uso de cada cenote como el límite de personas que un turista debería poder encontrar en estos espacios. 
Figura 6

\section{ESPEJO DE AGUA X’BATÚN}

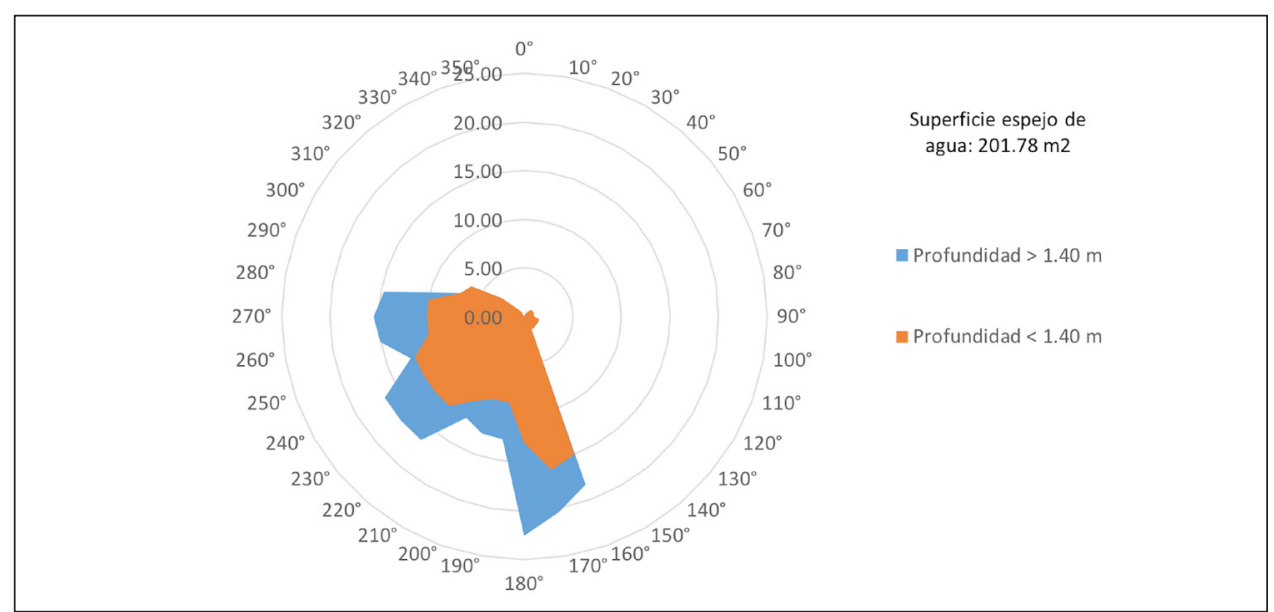

Fuente: Elaboración propia.

Figura 7

ESPEJO DE AGUA DZOMBAKAL

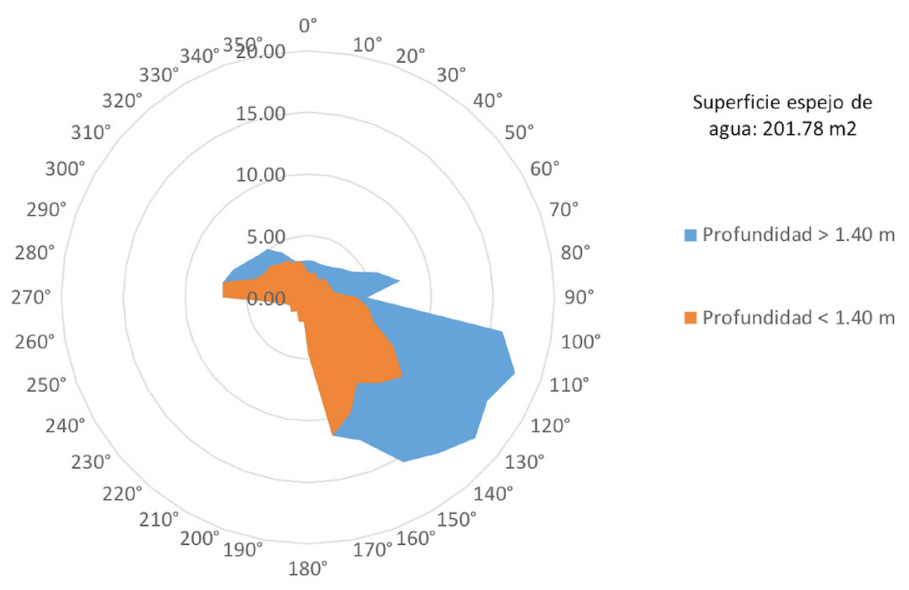

Fuente: Elaboración propia.

Para estimar la densidad máxima de uso (DMU), se dividió la superficie total de uso público de cada cenote entre $4 \mathrm{~m}^{2}$, que es el área vital estimada necesaria para que un visitante se sienta cómodo en espacios recreativos (García Hernández, 2001) (Formula 1 y Tabla 1). 


\section{Formula 1}

$$
D M U=\frac{E \Lambda+A D}{A V}
$$

DMU (Densidad máxima de uso)

EA (Espejo de agua)

AD (Área de Descanso)

AV (Área Vital)

Tabla 1

DENSIDAD MÁXIMA DE USO DE AMBOS CENOTES

\begin{tabular}{|l|c|c|c|c|}
\hline & $\begin{array}{c}\text { Superficie Espejo de agua } \\
\left(\mathbf{m}^{\mathbf{2}}\right)\end{array}$ & Área de descanso $\left(\mathbf{m}^{\mathbf{2}}\right)$ & Área vital $\left(\mathbf{m}^{\mathbf{2}}\right)$ & DMU $\left(\mathbf{m}^{\mathbf{2}}\right)$ \\
\hline $\mathrm{X}^{\prime}$ Batún & 283,33 & 36,6 & 4 & 80 \\
\hline Dzombakal & 201,78 & 18 & 4 & 55 \\
\hline
\end{tabular}

Fuente: Elaboración propia (La DMU fue redondeada para poder asignar el número máximo de personas en la fotografía final).

Así, la DMU fue de 80 personas para X’Batún y de 55 para Dzombakal. Estos números fueron los que se utilizaron en la fotografía número 6 de cada cenote. Las curvas de norma social (social norm curves) fueron construidas con los promedios de las respuestas sobre el nivel de aceptación que cada visitante asignó a las fotos. Se solicitó también a los visitantes que indiquen la foto que refleje mejor el número de personas que prefieren ver (Preferencia) y la foto que muestre mejor el número de personas que la cooperativa ecoturística debería dejar entrar al mismo tiempo en cada cenote (Management action).

\section{RESULTADOS}

Se respondieron un total de 189 cuestionarios en el cenote X’Batún y 192 en Dzombakal.

\subsection{Perfil del visitante}

De los 189 encuestados en X’Batún, el 56\% fueron residentes locales, es decir, del estado de Yucatán, principalmente de Mérida y las poblaciones cercanas; el 35\% fueron visitantes nacionales, considerados como todos los residentes de otros estados de México diferentes a Yucatán; y el 9\% visitantes extranjeros. La proporción más grande de personas que respondieron a la encuesta en X’Batún tienen una edad comprendida entre 18-29 años (40\%) seguida de aquellos que tienen entre 30-44 años (31\%). El grupo menos numeroso fue el de 60 años o más (4\%). Por otra parte, el $42 \%$ terminó una licenciatura universitaria (undergraduate studies) y el $11 \%$ posee un título de posgrado. 
De los 192 encuestados en Dzombakal, el 58\% fueron residentes locales, el 30\% fueron visitantes nacionales y el $12 \%$ visitantes extranjeros. La proporción más grande de encuestados tiene entre 18-29 años (34\%), seguida de aquellos que tienen entre 30-44 años (31\%). El grupo de menor tamaño fue el de aquellos con una edad de 60 años o más (6\%). Y en el ámbito intelectual, el 34\% terminó una licenciatura universitaria (undergraduate studies) y el $8 \%$ posee un título de posgrado.

En ambos cenotes, el $70 \%$ de los encuestados expresó que es la primera vez que los visita.

\subsection{Estándares de congestión para los dos cenotes}

Los hallazgos permitieron establecer estándares de congestión para ambos cenotes y confirman lo encontrado en estudios similares donde, en general, el nivel de aceptación experimentado por los visitantes disminuye conforme aumenta el número de personas presentes al mismo tiempo en un determinado espacio (Figura 8 y 9).

Figura 8

\section{CURVA DE NORMA SOCIAL DEL CENOTE X'BATÚN}

Figura 9

\section{CURVA DE NORMA SOCIAL DEL} CENOTE DZOMBAKAL

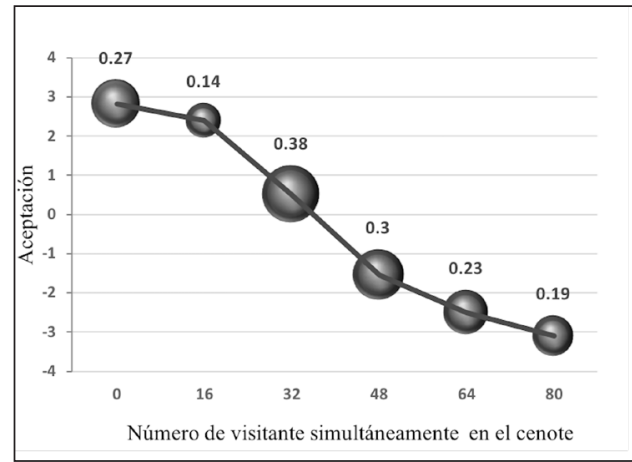

Fuente: Elaboración propia

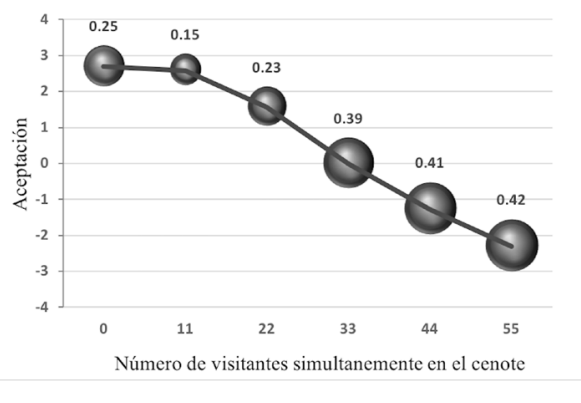

Fuente: Elaboración propia

Los visitantes de ambos cenotes manifestaron su nivel óptimo de aceptación cuando los cenotes están vacíos. El número máximo aceptado de personas presentes al mismo tiempo que un visitante está dispuesto a ver en X'batún es 37 mientras que en Dzombakal es 33. En promedio, todos los visitantes combinados, prefieren ver en X’batún hasta 15,7 personas y en Dzombakal 15,3 (Preferencia). En cuanto al promedio de personas que la cooperativa debería dejar entrar a los cenotes, los visitantes indicaron 32 para X’batún y 27,8 para Dzombakal (Management action). 


\subsection{Cristalización de la norma: PCI2}

Los valores del Índice Potencial de conflicto $\left(\mathrm{PCI}_{2}\right)$ fueron utilizados para estimar la cristalización de la norma (Vaske, J.J. y Whittaker, D., 2004). El rango de los valores de $\mathrm{PCI}_{2}$ varía de 0 a 1 , donde el 0 indica el más bajo nivel de conflicto y el 1 indica el nivel más alto de conflicto. Es decir, mientras más grande es el círculo, mayor es el nivel de conflicto y más bajo el nivel de consenso. Los círculos pequeños indican alto consenso y bajo conflicto. Los resultados de aplicar el $\mathrm{PCI}_{2}$ para cristalizar la norma arrojan un bajo nivel de conflicto en ambos cenotes, con variaciones ligeras de un cenote a otro. Para el caso de X'batún, los niveles de conflicto más altos se registran con 32 y 48 visitantes al mismo tiempo en el cenote. En Dzombakal, este nivel se manifiesta en los rangos bajos de aceptabilidad, es decir, con 33, 44 y 55 visitantes al mismo tiempo.

\subsection{Estándares de congestión entre visitantes locales, nacionales y extranjeros}

Como puede verse en las Figuras 10 y 11, el nivel de aceptación para los tres tipos de visitantes (locales, nacionales e internacionales) en ambos cenotes decrece conforme aumenta el número de personas. Tanto en el cenote $X^{\prime}$ batún como en Dzombakal la aceptación más baja la tuvieron los internacionales con 27 y 25 personas al mismo tiempo respectivamente. Le siguen los nacionales con 35 y 32 visitantes al mismo tiempo y por último los locales con la aceptación más alta: 40 visitantes en X’batún y 35.5 en Dzombakal.

Figura 10

\section{CURVA DE LA NORMA SOCIAL POR TIPO DE VISITANTE EN X'BATÚN}

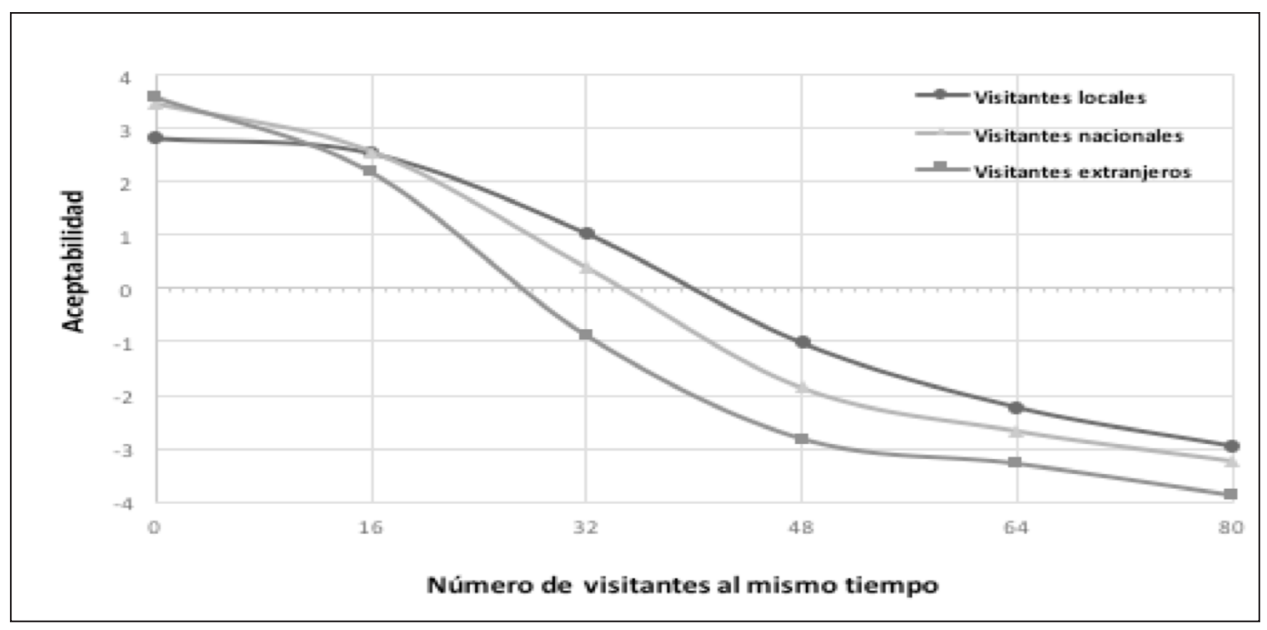

Fuente: Elaboración propia. 


\section{Figura 11 \\ CURVA DE LA NORMA SOCIAL POR TIPO DE VISITANTE EN DZOM- BAKAL}

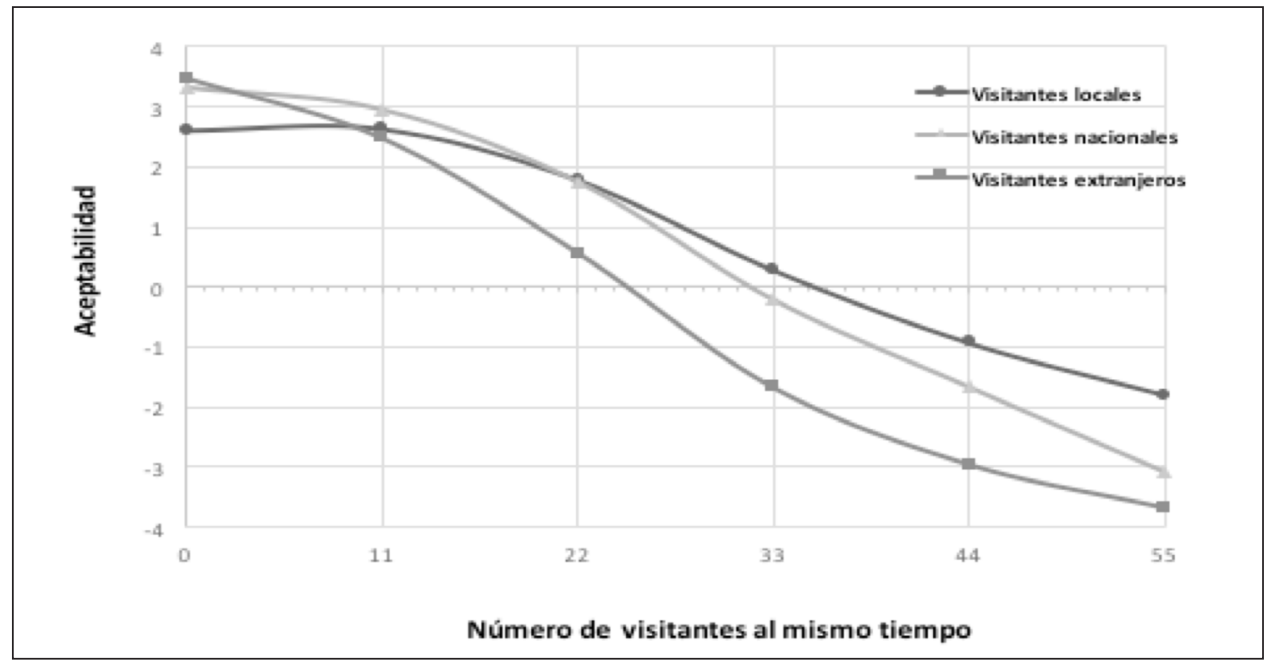

Fuente: Elaboración propia.

\section{DISCUSIÓN Y CONCLUSIÓN}

El gran reto para las cooperativas y otras organizaciones de la economía social, en cuanto a poner límites al uso del recurso natural sin menoscabo de sus ingresos, es encontrar el balance entre tarifas bajas y demanda alta o tarifas altas y demanda baja. El primero ejerce más presión sobre el recurso pero favorece el acceso a pobladores locales -en su mayoría de bajos y muy bajos ingresos- y desmotiva a visitantes nacionales y extranjeros que buscan sitios naturales con poca gente. El segundo ayuda a conservar el recurso, favorece la visita de ecoturistas nacionales y extranjeros pero desmotiva el acceso a locales por sus altas tarifas.

Los cenotes son cuevas inundadas localizadas en la península de Yucatán que forman la reserva hidrológica más grande de México. Su creciente uso turístico-recreativo así como los marcos normativos ambientales obligan a sus dueños y administradores, entre ellos, varias cooperativas ecoturísticas de campesinos, a realizar estudios de capacidad de carga de visitantes sin que exista todavía una metodología clara propuesta por las autoridades medioambientales.

Esta investigación propone una metodología para medir la dimensión social de la capacidad de carga en cenotes de la península de Yucatán y evaluar, así, los impactos negativos que la masificación tiene sobre la experiencia del visitante. Esta metodología, que ya ha sido aplicada en más de 181 sitios naturales y culturales, usa la teoría normativa y el método visual para determinar estándares de congestión de los visitantes según su origen, ya que muchas cooperativas cobran tarifas de entrada diferenciadas, según los visitantes 
sean locales (aquellos que viven en las poblaciones cercanas al cenote, incluyendo la ciudad de Mérida); nacionales (aquellos que residen en otros estados de México diferentes a Yucatán) o extranjeros (aquellos que vienen de otros países).

Se determinaron, así, los estándares de multitud de dos cenotes localizados en el Ejido de San Antonio Mulix, en la península de Yucatán: X’batún y Dzombakal. En ambos cenotes se encontró que la aceptación disminuye conforme aumenta el número de visitantes. En X’batún, el total de visitantes acepta ver no más de 37 personas al mismo tiempo, mientras que en Dzombakal la aceptación máxima es de 33, debido, en parte, a que la superficie de uso público en Dzombakal es más reducida que en X’batún.

El origen de los visitantes es uno de los factores sociodemográficos que puede afectar los estándares de multitud (Neuts y Nijkamp, 2012; Rasoolimanesh, Jaafar, Marzuki y Mohamad, 2016). Los resultados de este estudio muestran similitudes y diferencias entre los tres tipos de visitantes. Los visitantes internacionales son los que tienen el nivel de aceptación más bajo de entre los tres tipos y los locales son los que presentan los niveles de aceptación más altos. Estos resultados coinciden con los encontrados por Santiago, Gonzalez-Caban y Loomis (2008) y Sayan y Karagüzel (2010), que muestran que los estándares de multitud de visitantes locales puertorriqueños y turcos son más amplios que los mostrados por turistas extranjeros de países desarrollados. En los cenotes, esto podría deberse a factores culturales propios del mexicano de zonas rurales o proveniente de ciudades pequeñas y medianas que realiza actividades recreativas de una forma más colectivista, en grupos numerosos e incorporando a miembros de la familia extensa, mientras que los ciudadanos urbanos de países desarrollados practican la recreación en espacios naturales, sobre todo, en pareja, grupos pequeños o con miembros de la familia nuclear (Manning, 2011; Santiago et al., 2008; Sayan, Krymkowski, Manning, Valliere y Rovelstad, 2013). Sin embargo, estos resultados no coinciden con los expresados por Jin, Hu y Kavan (2016) donde visitantes de Europa y Norteamérica sienten menos congestión en el destino cultural Xi'an en China, que los visitantes chinos y japoneses.

De entre los tres tipos de visitantes, los internacionales son los que tienen los estándares más bajos respecto a la cantidad de personas que prefieren ver y el número máximo de personas que piensan que la cooperativa debería dejar entrar a los cenotes. Le siguen los nacionales y por último los locales. Estas diferencias entre tipos de visitantes podrían tener su origen también en factores culturales.

Los estándares de multitud encontrados en este estudio deben servir para mejorar el manejo de los cenotes de San Antonio Mulix ya que operativiza la dimensión social de la capacidad de carga de visitantes y la aplica a espacios recreativos con aguas confinadas. Los estándares de multitud son una alternativa efectiva y más simple de obtener por las cooperativas ecoturísticas que la capacidad de carga turística de Cifuentes (1992) que ha sido muy utilizada en áreas naturales protegidas de América Latina pero que presenta limitantes a la hora de adaptarla a cenotes. Los estándares aquí presentados deben ser tomados en cuenta por la cooperativa y, en caso de violación, aplicar las estrategias de gestión necesarias. Estas estrategias pueden ser desde limitar el número de visitantes hasta distribuirlos en el tiempo y en el espacio a través del establecimiento de bloques horarios de entrada que permita al cenote un flujo de visitantes adecuado sin llegar a la congestión (Enseñat-Soberanis, FraustoMartínez y Gándara-Vázquez, 2019). 
El reto de las cooperativas que administran los cenotes es lograr un balance entre la elitización del sitio reservado a turistas extranjeros que quieren ver un cenote con poca gente o vacío y la masificación del cenote ocasionado por visitantes locales que aceptan ver grandes cantidades de personas sin que esto afecte su experiencia.

Futuros estudios deben considerar la estimación de estándares de congestión en otros cenotes con características físicas diferentes a las de San Antonio Mulix y comparar resultados.

\section{BIBLIOGRAFÍA}

ALAZAIZEH, M.M., HALLO, J.C., BACKMAN, S.J., NORMAN, W.C. y VOGEL, M.A. (2015): «Crowding standards at Petra Archaeological Park: a comparative study of McKercher's five types of heritage tourists», Journal of Heritage Tourism, vol. 11 (4), pp. 364-381.

BEDDOWS, P., BLANCHON, P., ESCOBAR, E. y TORRES-TALAMANTE, O. (2007): «Los cenotes de la península de Yucatán», Arqueología mexicana, nº 83, pp. 32-35.

BLANCO GREGORY, R., ENSEÑAT SOBERANIS, F. y MONDRAGÓN MEJÍA, J.A. (2019): «La capacidad de carga psicosocial del turista: instrumento de medición para el desarrollo sostenible en la turistificación de los cenotes», Cuadernos de Turismo, $\mathrm{n}^{\circ} 43$, pp. 169-186.

BURNS, R. C., ARNBERGER, A. y VON RUSCHKOWSKI, E. (2010): «Social carrying capacity challenges in parks, forests, and protected areas : An examination of transatlantic methodologies and practices», International Journal of Sociology, vol. 40 (3), pp. 30-50.

CIFUENTES, M. (1992): Determinación de la Capacidad de Carga Turística en Áreas Protegidas. Informe Técnico. Turrialba, Costa Rica, CATIE, Programa de Manejo Integrado de Recursos Naturales.

COCCOSSIS, H. (2013): «Carrying Capacity as a tool for the Management of Tourism Pressures on Heritage Sites», en Agnew, N. y Demas, M. (Eds.) Visitor Management and Carrying Capacity at World Heritage Sites in China, Mogao Grottoes, China, The Getty Conservation Institute, pp. 35-38.

DRUMM, A. y MOORE, A. (2002): “An introduction to Ecotourism planning», en Ecotourism Development a Manual for Conservation Planners and Managers, vol.1

ENSEÑAT-SOBERANIS, F., FRAUSTO-MARTÍNEZ, O., GÁNDARA-VÁZQUEZ, M. (2019): A visitor flow management process for touristified archaeological sites, Journal of Heritage Tourism, vol. 14 (4), pp. 340-357.

GAONA-VIZCAYNO, S., GORDILLO DE ANDA, T. y VILLASUSO-PINO, M. (1980): «Cenotes, karst característico: mecanismos de formación», Revista Mexicana de Ciencias Geológicas, vol. 4 (3), pp. 32-36.

GARCÍA DE FUENTES, A., JOUAULT, S. y ROMERO, D. (2015): Atlas de Turismo Alternativo en la Península de Yucatán (1a ed.). Mérida, Cinvestav-Unidad Mérida.

GARCÍA HERNÁNDEZ, M. (2001): «Capacidad de acogida turística y gestión de flujos de visitantes en conjuntos monumentales: el caso de la Alhambra», PH: Boletín del Instituto Andaluz del Patrimonio Histórico, $\mathrm{n}^{\circ}$ 36, pp.124-137. 
GARCÍA HERNÁNDEZ, M., DE LA CALLE VAQUERO, M. y MÍNGUEZ GARCÍA, M.C. (2011): «Capacidad de carga turística y espacios patrimoniales. Aproximación a la estimación de la capacidad de carga del conjunto arqueológico de Carmona (Sevi1la, España)», Boletín de La Asociación de Geógrafos Españoles, n 57, pp. 219-241.

HEBERLEIN, T. A. \& VASKE, J. (1977): Crowding and visitor conflict on the Bois Brule River. Report WISC WRC 77-04. University of Wisconsin Water Resource Center. Madison, WI.

INEGI (1991): XI Censo General de Población y Vivienda. Mexico, D.F.

JACKSON, J. (1966): «A conceptual and measurement model for norms and roles», The Pacific Sociological Review, vol. 9 (1), pp. 35-47.

JIN, Q., HU, H. y KAVAN, P. (2016): «Factors influencing perceived crowding of tourists and sustainable tourism destination management», Sustainability, vol. 8 (10), pp. 1-17.

JOUAULT, S. (2018): Mayas d'hier et d'aujourd'hui: le rôle des sociétés locales dans le développement touristique. Rennes, Presses Universitaires de Rennes.

JOUAULT, S. \& JIMÉNEZ, M. (2015): «Región Cancún-Riviera Maya y su traspaís»,en A. García de Fuentes, S. Jouault, \& D. Romero (Eds.): Atlas de Turismo Alternativo en la Península de Yucatán. Mérida, Yucatán: Cinvestav-Unidad Mérida/UADY, pp. 124-128.

LEUNG, Y. F. \& MARION, J. L. (1999): «Characterizing backcountry camping impacts in Great Smoky Mountains National Park, USA», Journal of Environmental Management, vol. 57, n³, pp. 193-203.

MANNING, R. E. (2011): Studies in Outdoor Recreation, Search and Research for Satisfaction (3rd ed.). Corvallis, OR: Oregon State University Press.

MANNING, R. E. (2013): Parks and carrying capacity: Commons without tragedy. Washington, D.C.: Island Press.

MANNING, R. E., LIME, D., FREIMUND, W., \& PITT, D. (1996): «Crowding norms at frontcountry sites: A visual approach to setting standards of quality», Journal ofLeisure Sciences, no18, pp. 39-59.

MANNING, R. E. \& ANDERSON, L. E. (2012): Managing outdoors recreation: Case Studies in the National Parks. Wallingford, UK: CABI Publishing.

MCKERCHER, B. \& DU CROS, H. (2002): Cultural Tourism: The Partnership Between Tourism and Cultural Heritage Management. Londres: Haworth Hospitality Press. Retrieved from https://books.google.com.mx/books?id=InVGWVCI9F4C

NEUTS, B. \& NIJKAMP, P. (2012): « Tourist crowding perception and acceptability in cities. An Applied Modelling Study on Bruges», Annals of Tourism Research, vol. 39, $\mathrm{n}^{\mathrm{o}}$ 4, pp. 2133-2153. https://doi.org/10.1016/j.annals.2012.07.016

NEWSOME, D., DOWLING, R. K. \& MOORE, S. A. (2005): Wildlife tourism (Vol. 24). Channel View Publications.

RASOOLIMANESH, S. M., JAAFAR, M., MARZUKI, A. \& MOHAMAD, D. (2016): «How visitor and environmental characteristics influence perceived crowding», Asia Pacific Journal of Tourism Research, vol. 21, n 9, pp. 952-967. https://doi.org/10.10 80/10941665.2015.1084348

SANTIAGO, L. E., GONZALEZ-CABAN, A. \& LOOMIS, J. (2008): «A model for predicting daily peak visitation and implications for recreation management and water 
quality: evidence from two rivers in Puerto Rico», Environmental Management, vol. 41, nº 6, pp. 904-914.

SAYAN, S. \& KARAGÜZEL, O. (2010): «Problems of outdoor recreation: The effect of visitors' demographics on the perceptions of Termessos National Park, Turkey», Environmental Management, vol. 45, $\mathrm{n}^{\circ}$ 6, pp. 1257-1270.

SAYAN, S., KRYMKOWSKI, D. H., MANNING, R. E., VALLIERE, W. A.\& ROVELSTAD, E. L. (2013): Cultural influence on crowding norms in outdoor recreation: a comparative analysis of visitors to national parks in Turkey and the United States», Environmental Management, vol. 52, $\mathrm{n}^{\circ}$ 2, pp. 493-502.

SEDESOL (2013): Sistema de apoyo para la planeación del PDZP. Mexico, D.F.

SEDUMA (2014): Reglamento de la Ley de Protección al Medio Ambiente del Estado de Yucatán en Materia de Cenotes, Cuevas y Grutas. Pub. L. No. Decreto 193/2014, 1, 2014. México.

SEDUMA (2018): Oficio No. VI-1226-18. Mérida, Yucatán.

SEFOTUR (2017): Listado de cenotes con uso turístico. Mérida, Yucatán.

SHELBY, B. \& HEBERLEIN, T. A. (1986): Carrying capacity in recreation setting. Oregon State University Press.

SHELBY, B., VASKE, J. J. \& HARRIS, R. (1988): «User standards for ecological impacts at wilderness campsites», Journal of Leisure Research, $\mathrm{n}^{\circ}$ 20, pp. 245-256.

SHELBY, B., VASKE, J. J. \& HEBERLEIN, T. A. (1989): «Comparative analysis of crowding in multiple locations: Results from fifteen years of research», Leisure Sciences, vol. 11, n ${ }^{\circ} 4$, pp. 269-291.

TYLER, D. A., ROBERTS, D. W. \& NIELSEN, G. A. (1997): «Location and guidance for site-specific management», The state of site-specific management for agriculture, (thestateofsites), pp. 161-181.

VASKE, J. J. (2002): «Communicating judgments about practical significance: Effect size, confidence intervals and odds ratios», Human Dimensions of Wildlife, vol. 7, n 4, pp. 287-300.

VASKE, J. J. \& DONNELLY, M. P. (1988): «Normative evaluations of wildlife management». Paper presented at the Annual Congress of the National Recreation and Park Association, Indianapolis, Indiana.

VASKE, J. J. \& SHELBY, L. B. (2008): «Crowding as a descriptive indicator and an evaluative standard: Results from 30 years of research», Leisure Sciences, vol. 30, $\mathrm{n}^{\circ}$ 2, pp. 111-126.

VASKE, J. \& WHITTAKER, D. (2004): «Normative approaches to natural resources», in M. J. Manfredo, J. J. Vaske, B. L. Bruyere, \& D. R. Field (Eds.): Society and natural resources: A summary of knowledge. Jefferson, MO: Modern Litho, pp. 283-294.

WAGAR, J. A. (1964): «The Carrying Capacity of Wild Lands for Recreation», Forest Science, vol. 0(suppl_2), pp. a0001-24. Retrieved from http://dx.doi.org/10.1093/ forestscience/10.s2.a0001

WHITTAKER, D. \& SHELBY, B. (1992): «Developing good standards: Criteria, characteristics and sources», in B. Shelby, G. Stankey, \& B. Schindler, (Eds.): Defining wilderness quality: The role of standards in wilderness management - A workshop proceedings. Gen. Tech. Rep. PNW-GTR-305. U.S. Department of Agriculture, Forest Service, Pacific Northwest Research Station, p. 67. 